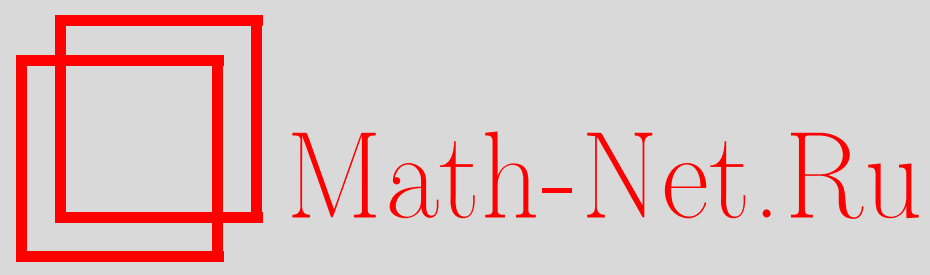

И. А. Юров, О p-адических функциях, сохраняющих меру Хаара, Матем. заметки, 1998, том 63, выпуск 6, 935-950

DOI: https://doi.org/10.4213/mzm1364

Использование Общероссийского математического портала Math-Net.Ru подразумевает, что вы прочитали и согласны с пользовательским соглашением http://www.mathnet.ru/rus/agreement

Параметры загрузки:

IP: 54.162 .85 .209

26 апреля 2023 г., $06: 28: 26$

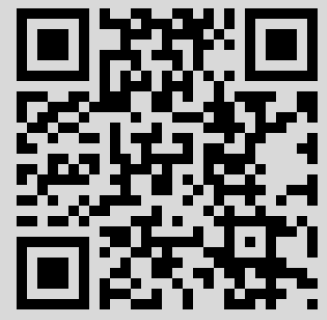




\title{
О p-АДИЧЕСКИХ ФУНКЦИЯХ, СОХРАНЯЮЩИХ МЕРУ ХААРА
}

\author{
И. А. Юров
}

Пусть $\left\{a_{n}\right\}_{n=0}^{\infty}-$ равномерно распределенная последовательность целых $p$-адических чисел. Статья посвящена изучению непрерьвных функций, близких к дифференцируемым в $p$-адической метрике: последовательность $\left\{f\left(a_{n}\right)\right\}_{n=0}^{\infty}$ равномерно распределена над кольцом целых $p$-адических чисел; последовательности $\left\{f_{k}\left(\varphi_{k}\left(a_{n}\right)\right)\right\}_{n=0}^{\infty}$ равномерно распределены над кольцом вычетов по $\bmod p^{k}$ для всех достаточно больших $k$, где $\varphi_{k}$ - канонический эпиморфизм кольца целых $p$-адических чисел на кольцо вычетов по $\bmod p^{k}$ и $f_{k}-$ функция, индуцированная $f$ накольце вычетов по $\bmod p^{k}$ (т.е. $\left.f_{k}(x)=f\left(\varphi_{k}(x)\right)\left(\bmod p^{k}\right)\right)$. Эти функции могут быть использованы, например, при построении генераторов псевдослучайньх чисел.

Библиограффия: 5 названий.

Пусть $f(x)$ - функция, заданная на множестве всех целых $p$-адических чисел $\mathbb{I}_{p}$, принимающая значения из этого множества, $\varphi_{k}: \mathbb{I}_{p} \rightarrow \mathbb{Z} / p^{k}-$ канонический эпиморфизм кольца $\mathbb{I}_{p}$ на кольцо вычетов $\mathbb{Z} / p^{k}$ по $\bmod p^{k}$, а $f_{k}(x)$ - функция, индуцированная $f(x)$ на $\mathbb{Z} / p^{k}\left(\right.$ т.е. $\left.f_{k}(x)=f\left(\varphi_{k}(x)\right)\left(\bmod p^{k}\right)\right)$. Пусть $\left\{a_{n}\right\}_{n=0}^{\infty}-$ равномерно распределенная последовательность цельх $p$-адических чисел.

Статья посвящена изучению непрерьвных функций, близких (в некотором точно определенном далее смысле) к дифференцируемым в $p$-адической метрике функциям: последовательность $\left\{f\left(a_{n}\right)\right\}_{n=0}^{\infty}$ равномерно распределена над $\mathbb{I}_{p} ;$ последовательности $\left\{f_{k}\left(\varphi_{k}\left(a_{n}\right)\right)\right\}_{n=0}^{\infty}$ равномерно распределены над $\mathbb{Z} / p^{k}$ для всех достаточно больших $k$. Эти функции могут быть использованы, например, при построении генераторов псевдослучайных чисел.

Дадим точные формулировки полученных результатов.

Пусть $A$ - пространство с заданной вероятностной мерой $\mu$ (например, $\mu$ - мера Хаaра). Напомним необходимые определения теории равномерно распределенных последовательностей, следуя [1].

В данной работе рассмотрены следуюшие случаи:

1) $A$ - компактная топологическая группа, изоморфная аддитивной групше кольца $\mathbb{I}_{p} ;$

2) $A$ - дискретная топологическая группа.

В случае 1 ) множества $a+p^{k} \mathbb{I}_{p}, k=1,2, \ldots, a \in \mathbb{I}_{p}$, образуют базу топологического пространства $A$, причем $\mu\left(a+p^{k} \mathbb{I}_{p}\right)=p^{-k}$. Последовательность $\left\{a_{n}\right\}_{n=0}^{\infty}, a_{n} \in \mathbb{I}_{p}$, назьвается равномерно распределенной над $\mathbb{I}_{p}(\mathrm{~cm} .[1])$, если

$$
\lim _{N \rightarrow \infty} \frac{1}{N} \nu_{N}\left(a+p^{k} \mathbb{I}_{p}\right)=p^{-k}, \quad k=1,2, \ldots, \quad a_{n} \in \mathbb{I}_{p}
$$


(здесь $\nu_{N}(U)$ - число тех $a_{0}, \ldots, a_{N}$, которые лежат в $U$ ).

В случае 2 ) каждое подмножество из $A$ является открытым и замкнутьм одновременно $\mu(U)=|U| \cdot|A|^{-1}$. Последовательность $\left\{a_{n}\right\}_{n=0}^{\infty}, a_{n} \in A$, назьвается равномерно распределенной, если

$$
\lim _{N \rightarrow \infty} \frac{1}{N} \nu_{N}(U)=\frac{|U|}{|A|}
$$

для каждого $U \subset A$.

ОПРЕДЕЛЕНИЕ 1 (см. [1]). Измеримая функция $f: A \rightarrow A$ (т.е. $f^{-1}(U) \mu$-измеримо при $\mu$-измеримом $U)$ сохраняет меру, если $\mu\left(f^{-1}(U)\right)=\mu(U)$ для каждого измеримого множества $U$.

Везде далее $f(x)$ - функция, заданная на $\mathbb{I}_{p}$, принимающая значения из этого множества.

ОПРЕДЕЛЕНИЕ 2 (см. [1]). Функция $f(x)$ асимптотически сохраняет меру, если существует натуральное число $B$ такое, что для любого $n \geqslant B$ функция $f_{n}$ биективна на $\mathbb{Z} / p^{k}$. Минимальное такое $B$ для данной $f$ обозначим через $B(f)$.

В дальнейшем будем говорить, что $f$ биективна по $\bmod p^{k}$, если $f_{k}$ биективна на $\mathbb{Z} / p^{k}$.

ОПРЕДЕЛЕНИЕ 3 (см. [2]). Функция $f(x)$ называется асимптотически консервативной, если существует натуральное число $P$ такое, что для любого $n \geqslant P$ из $a=b$ $\left(\bmod p^{n}\right)$ следует $f(a)=f(b)\left(\bmod p^{n}\right)$. Минимальное такое $P$ для данной $f$ обозначим через $P(f)$. Если $P(f)=1$, то будем говорить, что $f(x)$-консервативная функиия.

ОПРЕДЕЛЕНИЕ 4 (см. [2]). Функция $f(x)$ назьвается дифференцируемой по $\bmod p^{k} \boldsymbol{\theta}$ точке $u \in \mathbb{I}_{p}$, если существует натуральное число $N$ и $p$-адическое число $d_{k} f(u)$ такие, что для любых $n>N$ и $h \in \mathbb{I}_{p}$

$$
f\left(u+p^{n} h\right)=f(u)+p^{n} h d_{k} f(u)\left(\bmod p^{n+k}\right) .
$$

ОПРЕДЕЛЕНИЕ 5 (см. [2]). Функция $f(x)$ назьвается равномерно дифферениируемой no $\bmod p^{k}\left(\right.$ равномерно дифференцируемой по $\left.\bmod p^{n}\right)$, если существуют натуральное число $N$ и функция $d_{k} f(u)$, заданная на $\mathbb{I}_{p}$ и принимающая значения из поля $p$-адических чисел, такие, что для любых $n \geqslant N, h \in \mathbb{I}_{p}, x \in \mathbb{I}_{p}$ вьполняется соотношение (1). Минимальное такое $N$ для данной $f(x)$ обозначим через $N_{k}(f)$.

Функции, сохраняющие меру (асимптотически сохраняющие меру), позволяют из данной равномерно распределенной над $\mathbb{I}_{p}$ последовательности $\left\{a_{n}\right\}_{n=0}^{\infty}$ строить новые равномерно распределенные над $\mathbb{I}_{p}$ последовательности $\left\{f\left(a_{n}\right)\right\}_{n=0}^{\infty}$ (равномерно распределенные над $\mathbb{Z} / p^{k}$ последовательности $\left.\left\{f_{k}\left(\varphi_{k}\left(a_{n}\right)\right)\right\}_{n=0}^{\infty}, n=n_{0}, n_{0}+1, \ldots\right)[2$, предложение 1.2].

В работах [2], [3] изучались асимптотически консервативные функции, сохраняющие меру Хаара (асимптотически сохраняющие меру Хаара). В классе асимптотически консервативных равномерно дифференцируемых по $\bmod p$ функций понятия “функция $f$ сохраняет меру" и “функция $f$ асимптотически сохраняет меру" совпадают. В работах [2], [3] описаны сохраняющие меру Хаара функции в классе асимптотически консервативных равномерно дифференцируемых по $\bmod p$ функций. В частности, получено 
описание консервативных полиномиальных функций (т.е. заданных некоторым полиномом над полем $p$-адических чисел), сохраняющих меру Хаара.

В данной работе изучаются равномерно дифференцируемые по $\bmod p$ функции, сохраняющие меру Хаара или асимптотически сохраняющие меру Хаара, не являющиеся асимптотически консервативньми. Оказалось, что в этом случае понятия “функция $f$ сохраняет меру" и “функция $f$ асимптотически сохраняет меру" уже не совпадают.

Теорема 1 работы описьвает все равномерно дифференцируемые по $\bmod p$ функции $f: \mathbb{I}_{p} \rightarrow \mathbb{I}_{p}$, сохраняющие меру. А. А. Нечаев построил пример равномерно дифференцируемой по $\bmod p$, асимптотически сохраняющей меру функции, которая не является асимптотически консервативной. Именно, функция, заданная полиномом $\frac{1}{2}\left(x_{2}+x\right)$ при $p=2$, асимптотически сохраняет меру. Теорема 3 описьвает все равномерно дифференцируемые по $\bmod p$ функции $f: \mathbb{I}_{p} \rightarrow \mathbb{I}_{p}$, асимптотически сохраняющие меру, которые не являются асимптотически консервативными. Оказалось, что такие функции существуют лишь при $p=2$, а в классе полиномиальных функций приведенный вьше пример представляет собой в некотором смысле единственное исключение. Именно, если $f: \mathbb{I}_{p} \rightarrow \mathbb{I}_{p}-$ асимптотически сохраняющая меру функция, заданная полиномом над полем $p$-адических чисел, которая не является асимптотически консервативной функцией, то $p=2$ и $f(x)=V\left(\frac{1}{2}\left(x^{2}+x\right)\right)$, где $V: \mathbb{I}_{p} \rightarrow \mathbb{I}_{p}$ - асимптотически консервативная, сохраняющая меру функция, заданная полиномом над полем $p$-адических чисел.

1. Вспомогательные результаты. В этом пункте приведены вспомогательные утверждения, которые необходимы для доказательства основных результатов работы.

Через $\|\cdot\|_{p}$ обозначим $p$-адическую норму $p$-адического числа. Из предложения 2.12 работы [2] следует, что для равномерно дифференцируемой по $\bmod p^{k}$ функции $f$ величина $\left\|d_{k} f(x)\right\|_{p}$ ограничена на множестве $\mathbb{I}_{p}$. Через $s$ обозначим минимальное целое число такое, что $\left\|d_{k} f(x)\right\|_{p} \leqslant p^{s}, x \in \mathbb{I}_{p}$.

УТВЕРЖДЕНИЕ 1. Пусть функиия $f: \mathbb{I}_{p} \rightarrow \mathbb{I}_{p}$ равномерно дифференцируема по $\bmod p^{k} u\left\|d_{k} f(x)\right\|_{p} \geqslant 1$ для любого $x \in \mathbb{I}_{p}$. Тогда существует натуральное число $R \geqslant s$ такое, что для любого $i, 0 \leqslant i \leqslant p^{R}-1$, функиия $f_{i}(x)=f\left(i+p^{R} x\right)$ представима в виде $f_{i}(x)=c_{i}+p^{R-\varphi(i)} F_{i}(x)$, әде

1) $\left\|d_{k} f\left(i+p^{R} x\right)\right\|_{p}=p^{\varphi(i)}, \varphi(i) \geqslant 0$;

2) $0 \leqslant c_{i} \leqslant p^{R-\varphi(i)}-1$;

3) функиия $F_{i}: \mathbb{I}_{p} \rightarrow \mathbb{I}_{p}$ консервативна, равномерно дифференцируема по $\bmod p^{k+\varphi(i)}$ и асимптотически сохраняет меру, причем

$$
d_{k+\varphi(i)} F_{i}(x)=p^{\varphi(i)} d_{k} f(i)\left(\bmod p^{k+\varphi(i)}\right), \quad x \in \mathbb{I}_{p}, \quad N_{k+\varphi(i)}\left(F_{i}\right) \leqslant 1 .
$$

ДокАЗАТЕЛЬСТво. В случае $s=0$ из условия следует, что $\left\|d_{k} f(x)\right\|_{p}=1$ для любого $x \in \mathbb{I}_{p}$. В силу следствия 5.10 из [3] функция $f(x)$ не является асимптотически сохраняющей меру лишш потому, что $f(x)$ не биективна по $\bmod p^{N_{1}(f)}$. Как легко показать, существует функция $L: \mathbb{I}_{p} \rightarrow \mathbb{I}_{p}$ такая, что для любого $h \in \mathbb{I}_{p} L\left(x+p^{N_{1}(f)} h\right)=L(x)$ и функция $f+L$ биективна по $\bmod p^{N_{1}(f)}$, причем $N_{1}(f+L) \leqslant N_{1}(f)$. Пусть $R$ - натуральное число и $R \geqslant N_{k}(f)$. Для любого $i, 0 \leqslant i \leqslant p^{R}-1$, рассмотрим функцию $\bar{F}_{i}: \mathbb{I}_{p} \rightarrow \mathbb{I}_{p}$

$$
\bar{F}_{i}(x)=p^{-R}\left(f\left(i+p^{R} x\right)+L\left(i+p^{R} x\right)-f(i)-L(i)\right) .
$$


Легко видеть, что функция $\bar{F}_{i}$ равномерно дифференцируема по $\bmod p^{k}$, консервативна и асимптотически сохраняет меру. Тогда

$$
f\left(i+p^{R} x\right)=f(i)+L(i)-L\left(i+p^{R} x\right)+p^{R} \bar{F}_{i}(x) .
$$

Согласно выбору $R$ и определению функции $L$ имеем

$$
f\left(i+p^{R} x\right)=f(i)\left(\bmod p^{R}\right)+p^{R}\left(\bar{F}_{i}(x)+b_{i}\right), \quad \text { где } f(i)=f(i)\left(\bmod p^{R}\right)+p^{R} b_{i} .
$$

Свойства функции $F_{i}(x)=\bar{F}_{i}(x)+b_{i}$, перечисленные в 3$)$, имеют место при любом $b_{i} \in \mathbb{I}_{p}$. Для $s=0$ утверждение доказано.

В случае $s>0$ рассмотрим $r \geqslant \max \left\{N_{k}(f), s\right\}$. Пусть

$$
\begin{gathered}
\bar{F}_{i}(x)=p^{-(r-\varphi(i))}\left(f\left(i+p^{r} x\right)-f(x)\right), \quad i=0, \ldots, p^{r}-1 . \\
\left\|d_{k} f(i)\right\|_{p}=p^{\varphi(i)}, \quad s \geqslant \varphi(i) \geqslant 0,
\end{gathered}
$$

Легко показать, что функция $\bar{F}_{i}: \mathbb{I}_{p} \rightarrow \mathbb{I}_{p}$ консервативна, равномерно дифференцируема по $\bmod p^{k+\varphi(i)}$, причем $d_{k+\varphi(i)} \bar{F}_{i}(x)=p^{\varphi(i)} d_{k} f(i)\left(\bmod p^{k+\varphi(i)}\right), N_{k+\varphi(i)}\left(\bar{F}_{i}\right) \leqslant 1$. Если функция $\bar{F}_{i}$ не является асимптотически сохраняющей меру, то лишь потому, что

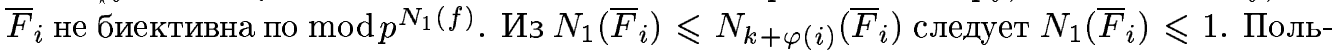
зуясь тем, что для $s=0$ утверждение доказано, представим функцию $\bar{F}_{i}(j+p x)$ в виде

$$
\bar{F}_{i}(j+p x)=c_{j}(x)+p F_{i, j}(x), \quad j=0, \ldots, p-1, \quad 0 \leqslant c_{j}(i) \leqslant p-1,
$$

где $F_{i, j}$ удовлетворяет условию 3$)$ утверждения. Тогда

$$
f\left(i+p^{r} j+p^{r+1} x\right)=f(i)+p^{r} c_{j}(i)+p^{r+1} F_{i, j}(x) .
$$

Для завершения доказательства осталось представить число $f(i)+p^{r} c_{j}(i)$ в виде $(f(i)+$ $\left.p^{r} c_{j}(i)\right)\left(\bmod p^{r+1}\right)+p^{r+1} b_{i, j}$ и заметить, что перечисленные в условии 3) свойства функции $F_{i, j}(x)$ сохраняются при добавлении к ней любой целой константы из $\mathbb{I}_{p}$. Утверждение доказано.

Введем дополнительные обозначения.

Положим $R(f)=1+\max \left\{N_{k}(f), s\right\}$. Пусть $R \geqslant R(f)$. Для $t=0, \ldots, p^{R}-1$ через $M_{j}(t)$ обозначим множество тех $i, 0 \leqslant i \leqslant p^{R}-1$, для которых $\varphi(i)=j\left(\left\|d_{k} f(i)\right\|_{p}=\right.$ $\left.p^{\varphi(i)}=p^{j}\right)$ и $f(i)=t\left(\bmod p^{R-j}\right)$. Пусть $r_{j}(t)=\left|M_{j}(t)\right|, M(t)=\bigcup_{j=0}^{s} M_{j}(t)$, $r(t)=|M(t)|$.

УТВЕРЖДЕНИЕ 2. Пусть функиия $f: \mathbb{I}_{p} \rightarrow \mathbb{I}_{p}$ равномерно дифференцируема по $\bmod p^{k} u 1 \leqslant\left\|d_{k} f(i)\right\|_{p} \leqslant p^{s}$. Функиия $f$ асимптотически сохраняет меру тогда $u$ только тогда, когда

1) $r_{0}(t) p^{s}+\cdots+r_{s}(t)=p^{s}$ для любого $t, 0 \leqslant t \leqslant p^{R}-1$;

2) величины $p^{-(n-s)}(f(x)-A)\left(\bmod p^{s}\right)$ образуют полную систему вычетов по $\bmod p^{s}$, в зависимости от того является ли $x, 0 \leqslant x \leqslant p^{n}-1$, решением сравнения $f(x)=A\left(\bmod p^{n-s}\right)$, для любых $n \geqslant R u A, 0 \leqslant A \leqslant p^{n-s}-1$. 
ДокаЗАТЕльСтво. Пусть $0 \leqslant A \leqslant p^{n-s}-1, A=t\left(\bmod p^{R}\right)$. Для любых $n \geqslant R$ и $i \in M_{j}(t)$ число решений сравнения

$$
f(x)=A\left(\bmod p^{n-s}\right)
$$

таких, что $x=i\left(\bmod p^{R}\right)$, равно $p^{s-j}$. Общее число решений сравнения $(2)$, лежащих в $\left\{0, \ldots, p^{n}-1\right\}$, равно $r_{0}(t) p^{s}+\cdots+r_{s}(t)=p^{s}$. Пусть $f$ - асимптотически сохраняющая меру функция. Тогда для любого $n \geqslant R \geqslant R(f) f(x)$ биективна по $\bmod p^{n}$ и, следовательно, $r_{0}(t) p^{s}+\cdots+r_{s}(t)=p^{s}$. Доказательство условия 2$)$ и доказательство утверждения 2 в обратную сторону опущены за очевидностью.

2. Равномерно дифференцируемые по $\bmod p$ функции, сохраняющие меру Хаара. В этом пункте приведены необходимые и достаточные условия того, что равномерно дифференцируемая по $\bmod p$ функция $f: \mathbb{I}_{p} \rightarrow \mathbb{I}_{p}$ сохраняет меру Хаара. Из этого критерия, в частности, следует, что в классе равномерно дифференцируемых по $\bmod p$, асимптотически консервативных функций $f: \mathbb{I}_{p} \rightarrow \mathbb{I}_{p}$ понятия " $f$ сохраняет меру" и " $f$ асимптотически сохраняет меру" совпадают.

ТЕОрема 1. Равномерно дифферениируемая функиия $f: \mathbb{I}_{p} \rightarrow \mathbb{I}_{p}$ сохраняет меру тогда и только тогда, когда

1) $\left\|d_{1} f(x)\right\|_{p} \geqslant 1$ для любого $x \in \mathbb{I}_{p}$;

2) для любого $t, 0 \leqslant t \leqslant p^{R}-1$, справедливо равенство

$$
r_{0}(t)+r_{1}(t) p^{-1}+\cdots+r_{s}(t) p^{-s}=1 .
$$

ДокаЗАтЕльство. Множества $T(a, k)=a+p^{k} \mathbb{I}_{p}, a=0, \ldots, p^{k}-1, k=1,2, \ldots,-$ база топологического пространства $x \in \mathbb{I}_{p}$. Обозначим через $\mu$ меру Хаарана $\mathbb{I}_{p}$. Тогда $\mu(T(a, k))=p^{-k}$. Покажем, что функция $f: \mathbb{I}_{p} \rightarrow \mathbb{I}_{p}$ сохраняет меру $\mu$ на $\mathbb{I}_{p}$ тогда и только тогда, когда

$$
\mu\left(f^{-1}(T(a, k))\right)=p^{-k}
$$

для любых $k \geqslant k_{0}, a=0, \ldots, p^{k}-1$, где $k_{0}$ - произвольное натуральное число. Для любого открытого подмножества $U$ из $\mathbb{I}_{p}$ равенство $\mu(U)=\mu\left(f^{-1}(U)\right)$ имеет место тогда и только тогда, когда вьполнено (4) для любых $k=1,2, \ldots, a=0, \ldots, p^{k}-1$. Действительно, пусть $U=\bigcup_{i \in J} T\left(a_{i}, k_{i}\right)$. Будем считать, что $a_{i} \neq a_{j}\left(\bmod p^{\nu}\right)$, $\nu=\min \left\{k_{i}, k_{j}\right\}$, для любых $i, j \in J, i \neq j\left(\right.$ если $a_{i}=a_{j}\left(\bmod p^{k_{i}}\right)$ и $k_{j} \geqslant k_{i}$, то $\left.T\left(a_{j}, k_{j}\right) \cup T\left(a_{i}, k_{i}\right)=T\left(a_{i}, k_{i}\right)\right)$. Тогда $f^{-1}(U)=\bigcup_{i \in J} f^{-1}\left(T\left(a_{i}, k_{i}\right)\right)$. Но для любых $i, j \in J, i \neq j$,

$$
f^{-1}\left(T\left(a_{i}, k_{i}\right)\right) \cap f^{-1}\left(T\left(a_{j}, k_{j}\right)\right)=\varnothing
$$

(если $x \in f^{-1}\left(T\left(a_{i}, k_{i}\right)\right) \cap f^{-1}\left(T\left(a_{j}, k_{j}\right)\right)$, то $f(x) \in T\left(a_{i}, k_{i}\right) \cap T\left(a_{j}, k_{j}\right)$, а это противоречит выбору $\left.a_{i}, a_{j}\right)$. Следовательно,

$$
\mu\left(f^{-1}(U)\right)=\sum_{i \in J} \mu\left(f^{-1}\left(T\left(a_{i}, k_{i}\right)\right)\right) .
$$

Однако, $T(a, w)=\bigcup_{i} T\left(a+p^{-w+k_{0}} i, k_{0}\right), i=0, \ldots, p^{-w+k_{0}}-1$, для любых $w=1, \ldots$, $k_{0}-1, a=0, \ldots, p^{w}-1$. Следовательно, функция $f$ сохраняет меру тогда и только тогда, когда $\mu\left(f^{-1}(T(a, k))\right)=p^{-k}$ для всех $k \geqslant k_{0}, a=0, \ldots, p^{k}-1$. 
Покажем, что если функция $f$ равномерно дифференцируема по $\bmod p$ и сохраняет меру $\mu$, то $\left\|d_{1} f(x)\right\|_{p} \geqslant 1$ для любого $x \in \mathbb{I}_{p}$. Действительно, пусть $u \in \mathbb{I}_{p}$ такое, что $\left\|d_{1} f(x)\right\|_{p} \leqslant 0$. Из равномерной дифференцируемости по $\bmod p$ функции $f$ следует, что для любого $h \in \mathbb{I}_{p}$ справедливо сравнение

$$
f\left(u+p^{n-1} h\right)=f(u)+d_{1} f(u) p^{n-1} h\left(\bmod p^{n}\right), \quad n \geqslant N_{1}(f) .
$$

Но $\left\|d_{1} f(x)\right\|_{p} \leqslant 0$, значит $f\left(u+p^{n-1} h\right)=f(u)\left(\bmod p^{n}\right)$ для любого $h \in \mathbb{I}_{p}$. Следовательно, $f^{-1}\left(f\left(u+p^{n} \mathbb{I}_{p}\right)\right) \supset u+p^{n-1} \mathbb{I}_{p}$ и

$$
\mu\left(f^{-1}\left(f\left(u+p^{n} \mathbb{I}_{p}\right)\right)\right) \geqslant \mu\left(u+p^{n-1} \mathbb{I}_{p}\right)=p^{-n+1},
$$

а по условию $\mu\left(f^{-1}\left(f\left(u+p^{n} \mathbb{I}_{p}\right)\right)\right)=p^{-n}$. Найдем $\mu\left(f^{-1}(T(a, n))\right)$, где $n \geqslant R \geqslant R(f)$. Пусть $K$-множество всех решений сравнений $f(x)=a\left(\bmod p^{n}\right), \operatorname{coдержащихся~в~}\{0, \ldots$, $\left.p^{n+s}-1\right\}$. Из равномерной дифференцируемости по $\bmod p$ функции $f$ следует, что для любого $h \in \mathbb{I}_{p} f\left(x_{0}+p^{n+s} h\right)=a\left(\bmod p^{n}\right), x_{0} \in K$. Тогда

$$
f^{-1}(T(a, n))=\bigcup_{x \in K} T(x, n+s) \quad \text { и } \quad \mu\left(f^{-1}(T(a, n))\right)=p^{-n-s}|K| .
$$

Через $\bar{a}$ обозначим наименьший неотрицательньй вычет числа $a$ по $\bmod p^{R}$. Покажем, что при $\left\|d_{1} f(x)\right\|_{p} \geqslant 1$ для любого $x \in \mathbb{I}_{p}$ выполняется соотношение $|K|=r_{s}(\bar{a})+\cdots$ $+r_{0}(\bar{a}) p^{s}$. Действительно, пусть $i \in M_{j}(\bar{a})$. Согласно утверждению 1

$$
f\left(i+p^{R} x\right)=c_{i}+p^{R-\varphi(i)} g_{i}(x), \quad c_{i}=\bar{a}\left(\bmod p^{R-\varphi(i)}\right),
$$

где функция $g: \mathbb{I}_{p} \rightarrow \mathbb{I}_{p}$ консервативна, равномерно дифференцируема по $\bmod p$ и асимптотически сохраняет меру $\mu$. Тогда число элементов множества $K$ таких, что $x_{i} \in K \cap$ $\left\{0, \ldots, p^{n-s}-1\right\}$ и $x_{i}=i\left(\bmod p^{R}\right)$, равно $p^{R-\varphi(i)}$. Таким образом,

$$
\mu\left(f^{-1}(T(a, n))\right)=p^{-n} \sum_{j=0}^{s} p^{-j} c_{i}(\bar{a})
$$

Следовательно, функция $f(x)$ сохраняет меру тогда и только тогда, когда $\left\|d_{1} f(x)\right\|_{p} \geqslant 1$ для любого $x \in \mathbb{I}_{p}$ и для любого $t, 0 \leqslant t \leqslant p^{R(f)}-1$, справедливо равенство (3). Теорема доказана.

СледСТВИЕ. Пусть равномерно дифферениируемая по $\bmod p$ функиия $f: \mathbb{I}_{p} \rightarrow \mathbb{I}_{p}$ асимптотически консервативна. Функиия $f$ сохраняет меру тогда и только тогда, когда $f$ асимптотически сохраняет меру.

Доказательство следует из критерия 5.4 работы [2] и теоремы 1.

3. Равномерно дифференцируемые по $\bmod p$, асимптотически сохраняющие меру Хаара функции, которые не являются асимптотически консервативными (редукционная теорема). Пункты 3,4 данной работы посвящены изучению равномерно дифференцируемых по $\bmod p$, асимптотически сохраняющих меру функций $f: \mathbb{I}_{p} \rightarrow \mathbb{I}_{p}$, которые не являются асимптотически консервативными. Основным результатом п. 3 является доказательство того, что равномерно дифференцируемые 
по $\bmod p$, асимптотически сохраняюшие меру функции $f: \mathbb{I}_{p} \rightarrow \mathbb{I}_{p}$, которые не являются асимптотически консервативными, существуют лиш при $p=2$.

Идея доказательства состоит в следующем.

1) Для каждой равномерно дифференцируемой по $\bmod p$ не являющейся асимптотически консервативной функции $f: \mathbb{I}_{p} \rightarrow \mathbb{I}_{p}$ строится некоторое аффинное многообразие $X$ над полем $\Omega_{p}\left(\Omega_{p}\right.$ есть алгебраическое замькание поля $p$-адических чисел).

2) Если $f(x)$ асимптотически сохраняет меру, то удается показать, что $X$ содержится в некоторой гиперплоскости аффинного пространства над полем $\Omega_{p}$ соответствующей размерности, а это оказьвается возможным лишш при $p=2$.

Введем дополнительные обозначения и примем некоторые соглашения о значениях параметров функции $f$.

Пусть функция $f: \mathbb{I}_{p} \rightarrow \mathbb{I}_{p}$ равномерно дифференцируема по $\bmod p$ и асимптотически сохраняет меру, $s$ - минимальное целое число такое, что $\left\|d_{1} f(x)\right\|_{p} \leqslant p^{s}, x \in \mathbb{I}_{p}$. Пусть $\left\|d_{1} f(x)\right\|_{p} \geqslant 1$ для любого $x \in \mathbb{I}_{p}$. Параметр $R$ выберем так, что $R \geqslant R(f)$ и для любого $n \geqslant R$ функция $f$ биективна по $\bmod p^{n}$. Пусть $0 \leqslant t \leqslant p^{R}-1$ и множество $M_{s}(t)$ непусто. Без ограничения общности будем считать, что $t=0$. Элементы множеств $M_{s}(t)$ и $M_{s-1}(t)$ будем обозначать числами от 1 до $r$ и от $r+1$ до $r+w$ соответственно.

Рассмотрим систему уравнений с неизвестными $\left(x_{1}, \ldots, x_{r+w}\right)$ над алгебраическим замьканием $\Omega_{p}$ поля $p$-адических чисел в случае $M_{s-1}(0) \neq \varnothing$ :

$$
\begin{aligned}
& f\left(1+p^{R} x_{1}\right)-f\left(2+p^{R} x_{2}\right)=0, \\
& f\left(1+p^{R} x_{1}\right)-f\left(r+p^{R} x_{r}\right)=0, \\
& f\left(1+p^{R} x_{1}\right)-f\left(r+w+p^{R} x_{r+w}\right)=0
\end{aligned}
$$

и систему уравнений с неизвестными $\left(x_{1}, \ldots, x_{r}\right)$ при $M_{s-1}(0)=\varnothing$ :

$$
\begin{aligned}
& f\left(1+p^{R} x_{1}\right)-f\left(2+p^{R} x_{2}\right)=0 \\
& \ldots \ldots \ldots \ldots \ldots \ldots \ldots \ldots \ldots \ldots \ldots \\
& f\left(1+p^{R} x_{1}\right)-f\left(r+p^{R} x_{r}\right)=0 .
\end{aligned}
$$

Будем говорить, что $\left(x_{1}, \ldots, x_{r+w}\right)$ - целое решение системы (5) (соответственно $\left(x_{1}, \ldots, x_{r}\right)$ - целое решение системы $\left.(6)\right)$, если каждая координата этого решения есть целое $p$-адическое число.

Рассмотрим случай $s \geqslant 2$. Пусть

$$
h_{i}= \begin{cases}p^{s} d_{k} f(i)\left(\bmod p^{2}\right) & \text { при } i=1, \ldots, r, \\ p^{s-1} d_{k} f(i)\left(\bmod p^{2}\right) & \text { при } i=r+1, \ldots, r+w .\end{cases}
$$

Через $a(x, h)$ и $b(x, h, d)$ обозначим функции с параметрами $h$ и $d, 0 \leqslant h \leqslant p^{2}-1$, $0 \leqslant d \leqslant p-1$, заданные на множестве целых $p$-адических чисел и принимающие значения из этого же множества:

$$
\begin{gathered}
a(x, h)=\left(x_{0} h\right)\left(\bmod p^{2}\right)+\left(x_{1} h\right)\left(\bmod p^{2}\right) p^{2}+\cdots \\
b(x, h, d)=\left(\left(x_{0}+d\right) h\right)\left(\bmod p^{2}\right)+\left(\left(x_{1}+d\right) h\right)\left(\bmod p^{2}\right) p^{2}+\cdots
\end{gathered}
$$

где $x \in \mathbb{I}_{p}, x=x_{0}+x_{1} p^{2}+x_{2} p^{4}+\cdots, 0 \leqslant x_{i} \leqslant p^{2}-1$. 
УТВЕРЖДЕНИЕ 3. Пусть равномерно дифферениируемая по $\bmod p$ функиия $f$ : $\mathbb{I}_{p} \rightarrow \mathbb{I}_{p}$ асимптотически сохраняет меру $u 1 \leqslant\left\|d_{1} f(x)\right\|_{p} \leqslant p^{s}, s \geqslant 2$. Тогда для достаточно больиого $R$

1) существуют единственное число $\tau, 0 \leqslant \tau \leqslant s$, и единственный набор чисел $\nu_{1}, \ldots, \nu_{r+w}$, дде $0 \leqslant \nu_{i} \leqslant p^{s-\tau}-1 n p u 1 \leqslant i \leqslant r, 0 \leqslant \nu_{i} \leqslant p^{s-\tau-1}-1$ при $r+1 \leqslant i \leqslant r+w$, такие, что на множсестве всех иельх решений $\left(x_{1}, \ldots, x_{r+w}\right)$ системы (5) выполняется соотношение

$$
\sum_{i=1}^{r} a\left(p^{\tau-s}\left(x_{i}-\nu_{i}\right), h_{i}\right)+\sum_{i=r+1}^{r+w} \sum_{j=0}^{p-1} b\left(p^{\tau-s+1}\left(x_{i}-\nu_{i}\right), h_{i}, j\right)=\frac{1}{2}(r+p w)=c_{1}
$$

2) существуют единственное число $\tau, 0 \leqslant \tau \leqslant s$, и единственный набор чисел $\nu_{1}, \ldots, \nu_{r}$, әде $0 \leqslant \nu_{i} \leqslant p^{s-\tau}-1$ при $i=1, \ldots, r$, такие, что на множестве всех иелых решений $\left(x_{1}, \ldots, x_{r}\right)$ системы (6) выполняется соотношение

$$
\sum_{i=1}^{r} a\left(p^{\tau-s}\left(x_{i}-\nu_{i}\right), h_{i}\right)=\frac{1}{2} r=c_{1}
$$

ДоКАЗАТЕЛЬСТВО. Рассмотрим систему уравнений

$$
f\left(1+p^{R} x_{1}\right)-f\left(i+p^{R} x_{i}\right)=0, \quad i \in M(0)=\{0, \ldots, m\}
$$

где $R \geqslant \max \{R(f), B(f)\}$. Пусть $\tau$ - минимальное натуральное число такое, что множество $M_{\tau}(0)$ непусто. Согласно утверждению 1

$$
f\left(i+p^{R} x_{i}\right)=p^{s-\varphi(i)} \Psi_{i}\left(x_{i}\right), \quad i \in M(0),
$$

где $\Psi_{i}(x)$ - равномерно дифференцируемая по $\bmod p^{1+\varphi(i)}$, консервативная, асимптотически сохраняющая меру функция, причем $N_{\varphi(i)+1}\left(\Psi_{i}\right) \leqslant 1$ и в соответствии с введенными обозначениями $\left\|d_{1} f(i)\right\|_{p}=p^{\varphi(i)}$. Тогда существует единственньй набор чисел $\nu_{i} \in\left\{0, \ldots, p^{\varphi(i)-\tau}-1\right\}$ такой, что $\Psi_{i}\left(\nu_{i}\right)=0\left(\bmod p^{\varphi(i)-\tau}\right)$. Это означает, что каждое уравнение системы $(7)$ принимает вид $\Psi_{1}\left(x_{1}\right)-p^{s-\varphi(i)} \Psi_{i}\left(x_{i}\right)=0$. Применяя утверждение 1 к функциям $\Psi_{i}$, получим $\Psi_{i}\left(\nu_{i}+p^{\varphi(i)-\tau} x_{i}\right)=p^{\varphi(i)-\tau} g_{i}\left(x_{i}\right)$, где $g_{i}$ - равномерно дифференцируемая по $\bmod p^{1+\varphi(i)}, i=1, \ldots, m$, консервативная, асимптотически сохраняющая меру функция. Таким образом, целые решения системы (7) представимы в виде $\left(\nu_{1}+p^{s-\tau} x_{1}, \nu_{2}+p^{\varphi(i)-\tau} x_{2}, \ldots, \nu_{m}+p^{\varphi(m)-\tau} x_{m}\right)$, где $m=|M(0)|$, и $\left(x_{1}, \ldots, x_{m}\right)-$ целое решение системы

$$
g_{1}\left(x_{1}\right)-g_{i}\left(x_{i}\right)=0, \quad i \in M(0) .
$$

Это означает, что доказательство условия 1 ) утверж дения 3 вытекает из того, что функция

$$
F_{1}\left(x_{1}, \ldots, x_{r+w}\right)=\sum_{i=1}^{r} a\left(x_{i}, h_{i}\right)+\sum_{i=r+1}^{r+w} \sum_{j=0}^{p-1} b\left(x_{i}, h_{i}, j\right)
$$

на множестве всех целых решений системы уравнений

$$
g_{1}\left(x_{1}\right)-g_{i}\left(x_{i}\right)=0, \quad i=2, \ldots, r+w,
$$


постоянна и ее значение равно $c_{1}$. Для любого $A=0, \ldots, p^{n}-1$ рассмотрим сравнение

$$
f(x)-p^{R-\tau} A=0\left(\bmod p^{n+R-\tau}\right), \quad x=0, \ldots, p^{n+R+s-\tau} .
$$

Пусть $x_{1}^{(n)}, 0 \leqslant x_{1}^{(n)} \leqslant p^{n}-1$, таково, что $f\left(1+p^{R}\left(\nu_{1}+p^{s-\tau} x_{1}^{(n)}\right)\right)-p^{R-\tau} A=0\left(\bmod p^{n}\right)$ (его существование следует из биективности $f$ по $\left.x_{1}^{(n)}\left(\bmod p^{n}\right)\right)$. Тогда, как легко убедиться, помимо $1+p^{R}\left(\nu_{1}+p^{s-\tau} x_{1}^{(n)}\right)$ сравнение (10) имеет решения вида $i+p^{R}\left(\nu_{i}+\right.$ $\left.p^{\varphi(i)-\tau}\left(x_{i}^{(n)}+p^{n} z_{i}\right)\right)$, где $0 \leqslant z_{i} \leqslant p^{s-\varphi(i)}-1 ;\left(x_{2}^{(n)}, \ldots, x_{m}^{(n)}\right)$ - целые решения системы сравнений $g_{1}\left(x_{1}^{(n)}\right)-g_{i}\left(x_{i}^{(n)}\right)=0\left(\bmod p^{n}\right), 0 \leqslant x_{i}^{(n)} \leqslant p^{n}-1$, а $x_{1}^{(n)}$ определено вьше. Согласно утверждению 2 числа

$$
\alpha\left(i, z_{i}, n\right)=p^{-(n+R-\tau)}\left(f\left(i+p^{R}\left(\nu_{i}+p^{\varphi(i)-\tau}\left(x_{i}^{(n)}+p^{n} z_{i}\right)\right)\right)-p^{R-\tau} A\right)\left(\bmod p^{s}\right)
$$

образуют полную систему вычетов по $\bmod p^{s}, i=1, \ldots, m, z_{i}=0, \ldots, p^{s-\varphi(i)}-1$. Это, в частности, означает, что сумма вычетов $\alpha\left(i, z_{i}, n\right)\left(\bmod p^{2}\right)$, как действительных чисел, равна $p^{s-2}\left(1+\cdots+p^{2}-1\right)$. С другой стороны,

$$
\begin{gathered}
\sum_{i, z_{i}} \alpha\left(i, z_{i}, n\right)\left(\bmod p^{2}\right)=\sum_{\substack{i \in M_{s}(0)\\
}} \alpha(i, n)\left(\bmod p^{2}\right)+\sum_{\substack{i \in M_{s-1}(0) \\
i \in M(0) \backslash\left(M_{s}(0) \cup M_{s-1}(0)\right) \\
z_{i}=0, \ldots, p^{s-\varphi(i)}-1}}^{p-1} \alpha\left(i, z_{i}, n\right)\left(\bmod p^{2}\right) .
\end{gathered}
$$

Из неравенства $1+\varphi(i) \geqslant 2$ следует, что функции $g_{i}(x)$ равномерно дифференцируемы по $\bmod p^{2}$, причем $N_{2}\left(g_{i}\right) \leqslant 1$. Тогда при любом $n \geqslant 1$ справедливы сравнения

$$
\begin{aligned}
p^{n+R-\tau} \alpha\left(i, z_{i}, n\right) & =f\left(i+p^{R}\left(\nu_{i}+p^{\varphi(i)-\tau}\left(x_{1}^{(n)}+p^{n} z_{i}\right)\right)\right)-p^{R-\tau} A \\
& =p^{R-\tau}\left(g_{i}\left(x_{i}^{(n)}\right)-A+p^{n} z_{i} d_{2} g_{i}\right)\left(\bmod p^{n+R+\tau+2}\right) .
\end{aligned}
$$

По выбору $x_{1}^{(n)} g_{i}\left(x_{i}^{(n)}\right)=A\left(\bmod p^{n}\right)$. Пусть $g_{i}\left(x_{i}^{(n)}\right)=A+p^{n} \beta_{i}(n)\left(\bmod p^{n+2}\right)$, где $0 \leqslant \beta_{i}(n) \leqslant p^{2}-1$. В соответствии с введенными обозначениями $p^{-\varphi(i)+s} d_{2} f(i)=d_{2} g_{i}$ $=h_{i}\left(\bmod p^{2}\right), i \in M(0)$. Справедливо сравнение

$$
\alpha\left(i, z_{i}, n\right)=\beta_{i}(n)+z_{i} h_{i}\left(\bmod p^{2}\right) .
$$

Из соотношений (11) и (12) получим

$$
\sum_{i, z_{i}} \alpha\left(i, z_{i}, n\right)\left(\bmod p^{2}\right)=\frac{1}{2}\left(p^{2}-1\right) p^{s} \sum_{i} p^{-\varphi(i)}=\frac{1}{2}\left(p^{2}-1\right) p^{s} \sum_{j=0}^{s-2} r_{j}(0) p^{-j}
$$

Из (12), (13) и условия 1) утверждения 2 получим

$$
\begin{aligned}
& \sum_{i \in M_{s}(0)}\left(c+\beta_{i}(n)\right)\left(\bmod p^{2}\right)+\sum_{i \in M_{s-1}(0)} \sum_{j=0}^{p-1}\left(c+\beta_{i}(n)+h_{i} j\right)\left(\bmod p^{2}\right) \\
& +\frac{1}{2}\left(p^{2}-1\right) p^{s} \sum_{j=0}^{s-2} r_{j}(0) p^{-j}=\frac{1}{2}\left(p^{2}-1\right) p^{s},
\end{aligned}
$$


где $0 \leqslant c \leqslant p^{2}-1$.

Найдем $\beta_{i}(n)$. Целые решения системы уравнений (8) представимы в виде

$$
x_{i}=x_{i}(0)+x_{i}(1) p^{2}+\cdots+x_{i}(k) p^{2 k}+\cdots, \quad \text { где } 0 \leqslant x_{i}(j) \leqslant p^{2}-1 .
$$

Пусть

$x_{i}^{(k+1)}=x_{i}(0)+x_{i}(1) p^{2}+\cdots+x_{i}(k+1) p^{2(k+1)}, \quad x_{i}^{(k)}=x_{i}(0)+x_{i}(1) p^{2}+\cdots+x_{i}(k) p^{2 k}$.

Так как функции $g_{i}(x)$ консервативны и $\left(x_{1}, \ldots, x_{m}\right)$ - целое решение системы $(8)$, справедливы сравнения

$$
g_{1}\left(x_{1}^{(k+1)}\right)-g_{i}\left(x_{1}^{(k+1)}\right)=0\left(\bmod p^{2(k+2)}\right), \quad g_{1}\left(x_{1}^{(k)}\right)-g_{i}\left(x_{1}^{(k)}\right)=0\left(\bmod p^{2(k+1)}\right) .
$$

Однако, $x_{i}^{(k+1)}=x_{i}^{(k)}+p^{2(k+1)} x_{i}(k+1)$. Тогда из равномерной дифференцируемости по $\bmod p^{2}$ функции $g_{i}(x)$ следует, что

$$
\beta_{i}(2 k)=\beta_{1}(2 k)+h_{1} x_{1}(k+1)-h_{i} x_{i}(k+1)=0\left(\bmod p^{2}\right) .
$$

Пусть $c=-\beta_{1}(2 k)-h_{1} x_{1}(k+1)$. Из (14) получим

$$
\sum_{i \in M_{s}(0)} h_{1} x_{i}(k+1)\left(\bmod p^{2}\right)+\sum_{i \in M_{s-1}(0)} \sum_{j=0}^{p-1} h_{i}\left(x_{i}(k+1)+j\right)\left(\bmod p^{2}\right)=\frac{1}{2}\left(1-p^{2}\right)(r+p w) .
$$

Умножим обе части равенства $(16)$ на $p^{2(k+1)}$ и просуммируем полученные уравнения по всем $k$. Тогда по определению функций $a(x, h)$ и $b(x, h, d)$

$$
\sum_{i \in M_{s}(0)} a\left(x_{i}, h_{i}\right)+\sum_{i \in M_{s-1}(0)} \sum_{j=0}^{p-1} b\left(x_{i}, h_{i}, j\right)=\frac{1}{2}(r+p w)=c_{1} .
$$

Тем самьп, завершается доказательство условия 1 ) утверждения 3 . Аналогично доказьвается условие 2) утверждения 3 , только в этом случае $w=0$ и $c_{2}=r / 2$.

Для случая $s=1$ рассмотрим функцию $e(x, h)$ с параметром $h, h=0, \ldots, p-1$, определенную на множестве всех целых $p$-адических чисел и принимающую значения из этого множества. Если $x \in \mathbb{I}_{p}$ и $x=x_{0}+x_{1} p+\cdots$, то

$$
e(x, h)=\left(x_{0} h\right)(\bmod p)+\left(x_{1} h\right)(\bmod p) p+\cdots .
$$

УТВЕРЖДЕНИЕ 4. Пусть равномерно дифферениируемая по $\bmod p$ функиия $f$ : $\mathbb{I}_{p} \rightarrow \mathbb{I}_{p}$ асимптотически сохраняет меру $u 1 \leqslant\left\|d_{1} f(x)\right\|_{p} \leqslant p^{s}$. Тогда при достаточно большом $R$

1) при $p \neq 2$ на множсестве всех иелых решений $\left(x_{0}, \ldots, x_{r}\right)$ системы (6) выполняется соотношение

$$
\sum_{i \in M_{s}(0)} e\left(x_{i}, h_{i}\right)=\frac{1}{2} p=c_{3}
$$

2) при $p=2$ на множестве всех иельх решений $\left(x_{1}, x_{2}\right)$ системы (6) выполняется соотношение $x_{1}+x_{2}+1=0$.

Доказывается в точности как утверждение 3 , только вместо функции $a(x, h)$ рассматривается $e(x, h)$ и в соотношениях $(11)-(16)$ вместо сравнимости по $\bmod p^{2}$ используется сравнимость по $\bmod p$, а $\tau=1$. В случае $p=2 e(x, h)=x\left(h_{i}=1(\bmod 2)\right)$. 
Теорема 2. Пусть равномерно дифферениируемая по $\bmod p$ функиия $f: \mathbb{I}_{p} \rightarrow \mathbb{I}_{p}$ не является асимптотически консервативной $u 1 \leqslant\left\|d_{1} f(x)\right\|_{p} \leqslant p^{s}$. Тогда если $f$ асимптотически сохраняет меру, то $p=2$ us $s=1$.

ДокаЗАТЕЛЬСТво. Как показано в утверждениях 3,4 , функции

$$
F_{1}=\sum_{i=1}^{r} a\left(x_{i}, h_{i}\right)+\sum_{i=r+1}^{r+w} \sum_{j=0}^{p-1} b\left(x_{i}, h_{i}, j\right), \quad F_{2}=\sum_{i=1}^{r} a\left(x_{i}, h_{i}\right), \quad F_{3}=\sum_{i=1}^{r} e\left(x_{i}, h_{i}\right)
$$

равны $c_{1}, c_{2}, c_{3}$ соответственно на множестве всех целых решений системы уравнений, состоящей из первых $r+w$ (или $r$ в случае 2 )) уравнений системы (8).

Рассмотрим случай $F_{1}\left(x_{1}, \ldots, x_{r+w}\right)=c_{1}$ на множествевсех целых решений системы уравнений (9). Всякое целое $p$-адическое число $x=x(0)+x(1) p^{2}+\cdots+x(k) p^{2 k}+\cdots$, где $0 \leqslant x(i) \leqslant p^{2}-1$, представим в виде

$$
\begin{gathered}
x=\sigma_{1}(x)+2 \sigma_{2}(x)+\cdots+\left(p^{2}-1\right) \sigma_{p^{2}-1}(x), \\
\sigma_{i}(x)=y_{i, 0}+p^{2} y_{i, 1}+\cdots+p^{2 k} y_{i, k}+\cdots, \quad y_{i, j}= \begin{cases}1 & \text { при } x(j)=i, i \neq 0, \\
0 & \text { в остальных случаях. }\end{cases}
\end{gathered}
$$

Тогда функция $F_{1}$ представима в виде

$$
F_{1}=\sum_{i=1}^{r} \sum_{j=1}^{p^{2}-1}\left(h_{i} j\left(\bmod p^{2}\right)\right) z_{i, j}+\sum_{i=r+1}^{r+w} \sum_{j=1}^{p^{2}-1} \sum_{k=0}^{p-1}\left(h_{i}(j+k)\left(\bmod p^{2}\right)\right) z_{i, j}
$$

где $z_{i, j}=\sigma_{j}\left(x_{i}\right)$. Обозначив коэффициенты при $z_{i, j}$ в $(17)$ через $a_{i, j}$, получим

$$
F_{1}=\sum_{i=1}^{r+w} \sum_{j=1}^{p^{2}-1} a_{i, j} z_{i, j}
$$

Пусть $C$ - множество всех целых решений системы уравнений

$$
\begin{gathered}
g_{1}\left(x_{1}\right)-g_{i}\left(x_{i}\right)=0, \quad i=2, \ldots, r+w \\
z_{i, j}=\sigma_{j}\left(x_{i}\right), \quad i=1, \ldots, r+w, \quad j=2, \ldots, p^{2}-1, \\
z_{i, 1}+\cdots+\left(p^{2}-1\right) z_{i, p^{2}-1}=x_{i}, \quad i=1, \ldots, r+w .
\end{gathered}
$$

Заметим, что соотношения (19) вьполняются как на множестве $C$, так и на алгебраическом замыкании этого множества. Под алгебраическим замыканием некоторого подмножества аффинного пространства над полем $\Omega_{p}$ понимается пересечение всех замкнутых множеств (т.е. множеств, которые состоят из всех решений системы полиномиальных уравнений над полем $\Omega_{p}$ ). Алгебраическое замыкание множества будем обозначать чертой сверху. Как легко видеть, для описания $\bar{C}$ достаточно изучить алгебраическое замькание $\bar{D}$ множества $D$ всех целых решений системы уравнений $(9),(18)$. Пусть $D_{i}$, $i=0, \ldots,\left(p^{2}-2\right)(r+w),-$ множество всех целых решений системы уравнений, состоящей из первых $r+w+i-1$ уравнений системы (9), (18). Пусть $q=\left(p^{2}-2\right)(r+w)$. Очевидно, что $\bar{D}_{0} \supset \cdots \supset \bar{D}_{q}=\bar{D}$. Покажем индукцией по $i$, что $\bar{D}_{0}=\bar{D}_{q}$. Пусть 
выполнено $\bar{D}_{0}=\cdots=\bar{D}_{i}$. Легко показать, что $\bar{D}_{i+1}$ совпадает с алгебраическим замыканием множества всех целых решений системы

$$
\begin{gathered}
g_{1}\left(x_{1}\right)-g_{i}\left(x_{i}\right)=0, \quad i=2, \ldots, r+w, \\
z_{\alpha, \beta}=\sigma_{\beta}\left(x_{\alpha}\right)
\end{gathered}
$$

при соответствующих значениях индексов $\alpha$ и $\beta$. Рассмотрим систему уравнений

$$
\begin{gathered}
g_{1}\left(x_{1}\right)-g_{i}\left(x_{i}\right)=0, \quad i=2, \ldots, r+w-1, \\
z_{\alpha, \beta}=\sigma_{\beta}\left(x_{\alpha}\right) .
\end{gathered}
$$

Если алгебраическое замькание множества всех цельх решений этой системы совпадает с алгебраическим замьканием множества всех целых решений системы

$$
g_{1}\left(x_{1}\right)-g_{i}\left(x_{i}\right)=0, \quad i=2, \ldots, r+w-1,
$$

то и алгебраическое замыкание множества всех целых решений системы (20) совпадает с алгебраическим замыканием множества всех целых решений системы (9). Значит, из предположения $\bar{D}_{i} \neq \bar{D}_{i+1}$ следует, что алгебраическое замькание множества всех целых решений уравнения $z_{\alpha, \beta}=\sigma_{\beta}\left(x_{\alpha}\right)$ не совпадает с аффинным пространством размерности 2 над полем $\Omega_{p}$. Следовательно, существует полином $U\left(z_{\alpha}, \beta, x_{\alpha}\right), \operatorname{deg} U \neq 0$, зависящий от двух переменных, который равен 0 на множестве всех целых решений уравнения $z_{\alpha, \beta}=\sigma_{\beta}\left(x_{\alpha}\right)$. Пусть $x_{\alpha}^{(0)} \in \mathbb{I}_{p}$ такое, что в последовательности $\left\{x_{\alpha}^{(0)}(i)\right\}_{i=0}^{\infty}$, где

$$
x_{\alpha}^{(0)}=x_{\alpha}^{(0)}(0)+x_{\alpha}^{(0)}(1) p^{2}+\cdots+x_{\alpha}^{(0)}(i) p^{2 i}+\cdots, \quad 0 \leqslant x_{\alpha}^{(0)}(i) \leqslant p^{2}-1,
$$

существует бесконечно много элементов $x_{\alpha}^{(0)}(i)$, которые не равны $\beta$. Тогда существует бесконечно много $x_{\alpha} \in \mathbb{I}_{p}$ таких, что $\sigma_{\beta}\left(x_{\alpha}\right)=\sigma_{\beta}\left(x_{\alpha}^{(0)}\right)$. Другими словами, полином $U\left(\sigma_{\beta}\left(x_{\alpha}^{(0)}\right), \sigma_{\beta}\left(x_{\alpha}\right)\right)$ имеет бесконечное число корней. Следовательно, переменная $z_{\alpha, \beta}$ принимает лиш конечное число значений из поля $\Omega_{p}$. Полученное противоречие показывает, что $\bar{D}_{i}=\bar{D}_{i+1}$. Таким образом, $\bar{D}_{0}=\bar{D}_{q}=\bar{D}$. Следовательно, $\bar{C}$ задано системой полиномиальных уравнений вида

$$
\begin{gathered}
x_{i}=z_{i, 1}+\cdots+\left(p^{2}-1\right) z_{i, p^{2}-1}, \quad i=1, \ldots, r+w, \\
u_{j}\left(x_{1}, \ldots, x_{r+w}\right)=0, \quad u_{j} \in \Omega_{p}\left[x_{1}, \ldots, x_{r+w}\right], \quad j \in J .
\end{gathered}
$$

Пересечение замкнутого множества $\bar{C}$ и гиперплоскости, заданной уравнением

$$
\sum_{i=1}^{r+w} \sum_{j=1}^{p^{2}-1} a_{i, j} z_{i, j}=c_{1}
$$

обозначим через $\bar{C}\left(F_{1}\right)$. Согласно утверждению 3 справедливо равенство $\bar{C}=\bar{C}\left(F_{1}\right)$. Пусть $\varphi$ - отображение $C$ (соответственно $\left.\bar{C}\left(F_{1}\right)\right)$ в аффинное пространство над полем $\Omega_{p}$ вида

$$
\varphi:\left(x_{1}, \ldots, x_{r+w}, z_{1,1}, \ldots, z_{r+w, p^{2}-1}\right) \mapsto\left(x_{1}, \ldots, x_{r+w}\right)
$$


Тогда слой над точкой $\left(x_{1}^{(0)}, \ldots, x_{r+w}^{(0)}\right)\left(\right.$ т.е. $\left.\varphi^{-1}\left(x_{1}^{(0)}, \ldots, x_{r+w}^{(0)}\right)\right)$ в $\bar{C}$ определяется системой уравнений

$$
z_{i, 1}+\cdots+\left(p^{2}-1\right) z_{i, p^{2}-1}=x_{i}^{(0)}, \quad i=1, \ldots, r+w
$$

а в $\bar{C}\left(F_{1}\right)$ - системой уравнений $(22),(21)$. Следовательно, существуют $b_{i} \in \Omega_{p}$ такие, что

$$
b_{i}=a_{i, 1}, \quad 2 b_{i}=a_{i, 2}, \ldots, \quad\left(p^{2}-1\right) b_{i}=a_{i, p^{2}-1}, \quad i=1, \ldots, r+w .
$$

Тогда для $i=1, \ldots, r$ справедливы соотношения

$$
h_{i}\left(\bmod p^{2}\right)=b_{i}, \ldots, \quad h_{i}\left(p^{2}-1\right)\left(\bmod p^{2}\right)=b_{i}\left(p^{2}-1\right)
$$

и для $i=r+1, \ldots, r+w$

$$
\sum_{k=0}^{p-1}\left((1+k) h_{i}\right)\left(\bmod p^{2}\right)=b_{i}, \quad \ldots, \sum_{k=0}^{p-1}\left(\left(p^{2}-1+k\right) h_{i}\right)\left(\bmod p^{2}\right)=b_{i}\left(p^{2}-1\right)
$$

Тогда $h_{i}\left(\bmod p^{2}\right)=1$ для $i=1, \ldots, r$, а для $i=r+1, \ldots, r+w$

$$
j \sum_{k=0}^{p-1}\left((k+1) h_{i}\right)\left(\bmod p^{2}\right)=\sum_{k=0}^{p-1}\left((j+k) h_{i}\right)\left(\bmod p^{2}\right), \quad j=2, \ldots, p^{2}-1 .
$$

Если множество $M_{s-1}(0)$ непусто (т.е. $w \neq 0$ ), то из полученного выше противоречия следует, что не существует равномерно дифференцируемых по $\bmod p$, асимптотически сохраняющих меру функций, которые не являются асимптотически консервативными. Если же $M_{s-1}(0)=\varnothing($ т.е. $w=0)$, то $h_{i}\left(\bmod p^{2}\right)=1$ для $i=1, \ldots, r$. Пусть $a \in \mathbb{I}_{p},\|a\|_{p}=1,\|a-1\|_{p}=1$. Согласно выбору $a$ соотношения $a h_{i}\left(\bmod p^{2}\right)=1$ для $i=1, \ldots, r\left(p^{\varphi(i)} d_{1} a f(x)=a h_{i}\left(\bmod p^{2}\right)\right)$, соответствующие функции $a f(x)$, не выполняются и, следовательно, функция $f(x)$ не является асимптотически сохраняющей меру. Случай $p \neq 2, s=1$, соответствующий функции $F_{3}$, доказьвается аналогично, только вместо сравнения по $\bmod p^{2}$ используется сравнение по $\bmod p$. Tеорема доказана.

4. Равномерно дифференцируемые по $\bmod p$, асимптотически сохраняющие меру Хаара функции, которые не являются асимптотически консервативными (основные результаты). В этом пункте описаны равномерно дифференцируемые по $\bmod p$, асимптотически сохраняющие меру функции $f: \mathbb{I}_{p} \rightarrow \mathbb{I}_{p}$, которые не являются асимптотически консервативными. Результаты этого пункта и соответствующие результаты работ [2], [3] описывают все равномерно дифференцируемые по $\bmod p$ функции $f: \mathbb{I}_{p} \rightarrow \mathbb{I}_{p}$, асимптотически сохраняющие меру. В частности, описаны все полиномы над полем $p$-адических чисел, которые задают асимптотически сохраняющую меру функцию, отображающую кольцо цельх $p$-адических чисел в себя.

Пусть $G$ - множество 2-адических функций, которые имеют следующее строение. Зафиксируем некоторое натуральное $R, R \geqslant 1$. Множество $\left\{0, \ldots, 2^{R}-1\right\}$ разобьем на два непересекающихся подмножества $S_{1}$ и $S_{0}\left(S_{0}\right.$ может быть пустым). Множества $S_{1}$ и $S_{0}$ разобьем на двухэлементные непересекающиеся подмножества $S_{1}(1), \ldots, S_{1}(w)$ и $S_{0}(1), \ldots, S_{0}\left(2^{R-1}-w\right)$. Пусть $\psi$ - произвольное отображение $S_{1}$ в $\left\{0, \ldots, 2^{R-1}-1\right\}$ 
такое, что $\psi(i)=\psi(j)$ при $i, j \in S_{1}(\alpha), i \neq j$ и и $\psi(i) \neq \psi(j)$ при $i \in S_{1}(\alpha), j \in S_{1}(\beta)$, $\alpha \neq \beta$. Пусть $\varphi$ - произвольное отображение $S_{0}$ в $\left\{0, \ldots, 2^{R-1}-1\right\} \backslash \psi\left(S_{1}\right)$, обладающее теми же свойствами, что и $\psi$. Функция из $G$ имеет вид

$$
\begin{array}{ll}
f\left(i+2^{R} x\right) & =\psi(i)+2^{R} g_{i}(x), \\
f\left(j+2^{R} x\right) & =\psi(i)+2^{R} g_{i}(-1-x), \\
f\left(i+2^{R} x\right) & =\varphi(i)+2^{R-1} q_{\alpha}+2^{R} g_{i}(x), \\
f\left(j+2^{R} x\right) & =\varphi(i)+2^{R-1}\left(1-q_{\alpha}\right)+2^{R} g_{j}(x),
\end{array}
$$

где $q_{\alpha}=0,1, g_{i}: \mathbb{I}_{2} \rightarrow \mathbb{I}_{2}-$ консервативные, сохраняющие меру функции, причем $g_{i}(x)$ равномерно дифференцируема по $\bmod 2^{2}, N_{2}\left(g_{i}\right) \leqslant 1$ при $i \in S_{1}$ и $g_{i}(x)$ равномерно дифференцируема по $\bmod 2, N_{1}\left(g_{i}\right) \leqslant 1$ при $i \in S_{0}$.

ТЕОрема 3. Пусть равномерно дифференцируемая по $\bmod p$ функиия $f: \mathbb{I}_{p} \rightarrow \mathbb{I}_{p}$ не является асимптотически консервативной функиией. Функиия $f$ асимптотически сохраняет меру тогда и только тогда, когда $p=2 u f \in G$.

ДокАЗАТЕЛЬСтво. Пусть $f(x)$ асимптотически сохраняет меру. Из теоремы 2 и предложения 5.2 из [3] следует, что $p=2$ и $1 \leqslant\left\|d_{1} f(x)\right\|_{2} \leqslant 2$. Из утверждения 1 следует, что для достаточно большого $R$ функция $f\left(i+2^{R} x\right)$ совпадает с функцией $c_{i}+2^{R-\varphi(i)} g_{i}(x)$, где $g_{i}: \mathbb{I}_{2} \rightarrow \mathbb{I}_{2}$ удовлетворяют условию 3$)$ этого утверждения. Пусть $i, j, 0 \leqslant i, j \leqslant 2^{R}-1$, такие, что $\left\|d_{1} f(i)\right\|_{2}=\left\|d_{1} f(j)\right\|_{2}$ и $c_{i}=c_{j}\left(\bmod 2^{R-1}\right)$. Из утверждения 4 следует, что $\bar{C}\left(C\right.$ - множество всех целых решений уравнения $g_{i}(x)-$ $\left.g_{j}(y)=0\right)$ совпадает с многообразием $L=\left\{(x, y) \mid x, y \in \Omega_{p}, x+y+1=0\right\}$. Действительно, $\bar{C} \subset L$ и $\operatorname{dim}_{\Omega_{p}} \bar{C}=1$. Пусть $X_{1}$ - многообразие такое, что $X_{1} \subset \bar{C}$ и $\operatorname{dim}_{\Omega_{p}} X_{1}=1$. Тогда из $X_{1} \subset \bar{C} \subset L$ в силу теоремы 1 из [4, §6, гл. 1] (см. также [5, гл. $1, \S 1$, упражнение 1.10]) имеем $X_{1}=\bar{C}=L$. Таким образом, $g_{i}(x)=g_{j}(-x-1)$ для любого $x \in \mathbb{I}_{p}$. Это доказьвает теорему в одну сторону.

Для доказательства теоремы в обратную сторону необходимо показать, что функция $f \in G$ равномерно дифференцируема по $\bmod 2$ и асимптотически сохраняет меру. Из равномерной дифференцируемости по $\bmod 2$ функции $g_{i}(x)$ при $i \in S_{0}\left(\right.$ по $\bmod 2^{2}$ при $i \in S_{1}$ ) следует равномерная дифференцируемость по $\bmod 2$ функции $f$. Пусть $0 \leqslant A \leqslant 2^{n+R}-1$. Покажем, что для любого натурального $n$ существует единственное $x, 0 \leqslant x \leqslant 2^{n+R}-1$, такое, что $f(x)=A\left(\bmod 2^{n+R}\right)$. Так как $\psi\left(S_{1}\right) \cup \varphi\left(S_{0}\right)=\{0$, $\left.\ldots, 2^{R-1}-1\right\}$ и $\psi\left(S_{1}\right) \cap \varphi\left(S_{0}\right)=\varnothing$, найдется единственноемножество $S_{1}(\alpha)$ или $S_{0}(\alpha)$ такое, что для его элементов $u_{1}, u_{2}$ справедливы сравнения $\varphi\left(u_{1}\right)=A=\varphi\left(u_{2}\right)\left(\bmod 2^{R-1}\right)$ либо $\psi\left(u_{1}\right)=A=\psi\left(u_{2}\right)\left(\bmod 2^{R-1}\right)$.

Рассмотрим случай $\left\{u_{1}, u_{2}\right\}=S_{0}(\alpha)$. Пусть $u_{1}$ такое, что $f\left(u_{1}\right)=A\left(\bmod 2^{R}\right)$. Тогда $f\left(u_{2}\right) \neq A\left(\bmod 2^{R}\right)$. Действительно,

$$
f\left(u_{1}\right)=\varphi\left(u_{1}\right)+2^{R-1} q_{\alpha}\left(\bmod 2^{R}\right), \quad f\left(u_{2}\right)=\varphi\left(u_{2}\right)+2^{R-1}\left(1-q_{\alpha}\right)\left(\bmod 2^{R}\right) .
$$

Но $f\left(u_{1}+2^{R} x\right)=A\left(\bmod 2^{R}\right)+2^{R} g_{u_{1}}(x)$ для единственного $u_{1}, 0 \leqslant u_{1} \leqslant 2^{R}-1$. В силу биективности функции $g_{u_{1}}(x)$ по $\bmod 2^{n}$ существует единственное $x_{0}$ такое, что $g_{u_{1}}\left(x_{0}\right)=2^{-R}\left(A-A\left(\bmod 2^{R}\right)\right)\left(\bmod 2^{n}\right)$.

Рассмотрим случай $\left\{u_{1}, u_{2}\right\}=S_{1}(\alpha)$. Так как $u_{1}$ и $u_{2}$ таковы, что $f\left(u_{1}\right)=f\left(u_{2}\right)=A$ $\left(\bmod 2^{R-1}\right)$, условие 1$)$ утверждения 2 выполнено. По условию

$f\left(u_{1}+2^{R} x\right)=A\left(\bmod 2^{R-1}\right)+2^{R-1} g_{u_{1}}(x), \quad f\left(u_{2}+2^{R} x\right)=A\left(\bmod 2^{R-1}\right)+2^{R-1} g_{u_{2}}(x)$, 
где $g_{u_{1}}(x)$ и $g_{u_{2}}(x)$ - сохраняющие меру, консервативные функции. Следовательно, найдутся единственные $x, y, 0 \leqslant x, y \leqslant 2^{n}-1$, такие, что

$$
f\left(u_{1}+2^{R} x\right)=f\left(u_{2}+2^{R} y\right)\left(\bmod 2^{n+R}\right) .
$$

Пусть $y=2^{n+1}-1-x$. Справедливы сравнения

$$
\begin{aligned}
& f\left(u_{1}+2^{R} x\right)-f\left(u_{2}+2^{R} y\right)=2^{R-1}\left(g_{u_{1}}(x)-g_{u_{2}}\left(2^{n+1}-1-x\right)\right) \\
& \quad=2^{R-1}\left(g_{u_{1}}(x)-g_{u_{2}}(-x-1)-2^{n+1} d_{2} g_{u_{2}}(x)\right)\left(\bmod 2^{n+R+1}\right) .
\end{aligned}
$$

По условию $g_{u_{1}}(x)=g_{u_{2}}(-x-1)$ и $\left\|d_{2} g_{u_{2}}(x)\right\|_{2}=1$. Следовательно, $f\left(u_{1}+2^{R} x\right)-$ $f\left(u_{2}+2^{R} y\right)=2^{n+R}\left(\bmod 2^{n+R+1}\right)$. Тогда из утверждения 2 следует, что функция $f$ асимптотически сохраняет меру. Теорема доказана.

Пусть $f(x)$ - целозначньй полином над полем $p$-адических чисел (т.е. задаваемая им функция отображает кольцо $\mathbb{I}_{p}$ в себя).

ТЕОРемА 4. Полином $f(x)$ асимптотически сохраняет меру тогда и только тогда, когда либо $f(x)$ - асимптотически консервативный и сохраняющий меру полином, либо $p=2$ u $f(x)=V\left(\frac{1}{2}\left(x^{2}+x\right)\right)$, где $V(y)$ - челозначный асимптотически консервативный полином, сохраняюший меру.

ДокАЗАТЕльство. Легко видеть, что функция, соответствующая полиному $f(x)$, равномерно дифференцируема по $\bmod p$. Если $f(x)$ - асимптотически консервативньй полином, то утверждение теоремы вытекает из следствия теоремы 1 . Если $f(x)$ - асимптотически сохраняющий меру полином, который не является асимптотически консервативным, то по теореме 2 и предложению 5.2 из [3] $p=2$ и $1 \leqslant\left\|d_{1} f(x)\right\|_{2} \leqslant 2, x \in \mathbb{I}_{2}$. Согласно условию 2) утверждения 4 значение $x_{1}+x_{2}+1$ равно 0 на множестве всех целых решений уравнения $f\left(a+2^{R} x_{1}\right)-f\left(b+2^{R} x_{2}\right)=0$ для подходящих $a, b$ и $R$. Напомним, что в рассматриваемом случае $0 \leqslant a, b \leqslant 2^{R}-1$. Для любого $x_{1} \in \mathbb{I}_{2}$ справедливо соотношение $f\left(a+2^{R} x_{1}\right)-f\left(b+2^{R}\left(-x_{1}-1\right)\right)=0$. Пусть $u=a+2^{R} x_{1}$, $v=b+2^{R} x_{2}, d=a+b-2^{R}$. Заметим, что

$$
f(u)=\bar{f}_{0}(0)+(u(d-u))\left(\bar{f}_{1}(0)+(u(d-u))\left(\bar{f}_{2}(0)+\left(\ldots(u(d-u)) \bar{f}_{k}(0) \ldots\right)\right)\right),
$$

где $\bar{f}_{k}(u)-\bar{f}_{k}(0)=u(d-u) \bar{f}_{k+1}(u), k=0, \ldots, \frac{1}{2} \operatorname{deg} f(u), \bar{f}_{0}(u)=f(u)$. Следовательно, $f(u)=G(u(d-u))$, где $G(y)=\bar{f}_{0}(0)+\cdots+\bar{f}_{k} y^{k}$. Если композищия функций $H_{1}: \mathbb{I}_{p} \rightarrow \mathbb{I}_{p}$ и $H_{2}: \mathbb{I}_{p} \rightarrow \mathbb{I}_{p}$ асимптотически сохраняет меру, то функции $H_{1}$ и $H_{2}$ также асимптотически сохраняют меру. Действительно,

$$
\left|H_{1}\left(H_{2}\left(\left\{0, \ldots, p^{n}-1\right\}\right)\right)\right| \leqslant \min \left\{\left|H_{1}\left(\left\{0, \ldots, p^{n}-1\right\}\right)\right|,\left|H_{2}\left(\left\{0, \ldots, p^{n}-1\right\}\right)\right|\right\}
$$

для любого достаточно большого $n$.

Рассмотрим случаи $d=0(\bmod 2)$ и $d=1(\bmod 2)$. В первом случае согласно следствию 5.10 из [3] полином $u(d-u)$ не является асимптотически сохраняющим меру.

Пусть $d=1(\bmod 2)$ и $f(u)=V\left(\frac{1}{2}\left(u^{2}-d u\right)\right)$, где $V(y)=G(-2 y)$. Так как $f$ асимптотически сохраняет меру, то, как отмечалось выше, полиномы $V(y)$ и $\frac{1}{2}\left(u^{2}-d u\right)$ асимптотически сохраняют меру. Однако, $\left\|d_{1} f(u)\right\|_{2} \leqslant 2$ и $\left\|d_{1} \frac{1}{2}\left(u^{2}-d u\right)\right\|_{2}=2$, где $u \in \mathbb{I}_{2}$. В силу следствия 5.10 из [3] справедливо равенство $\left\|d_{1} V(y)\right\|_{2}=1$ и, следовательно, $V(y)$ - целозначньй, асимптотически консервативньй полином, сохраняющий меру. 
Пусть полином $T_{d}(u)=\frac{1}{2}\left(u^{2}-d u\right)$ асимптотически сохраняет меру. Напомним, что $d=a+b-2^{R}, 0 \leqslant a, b \leqslant 2^{R}-1$. Из условия $d=1(\bmod 2)$ следует, что $d=1+2 q$, где $q=-2^{R-1},-2^{R-1}+1, \ldots, 2^{R-1}+2$.

Если $q \geqslant 0$, то $d>0$ и полином $T_{d}(u)$ имеет два различных корня на множестве чисел $\{0,1,2, \ldots\}$. Это противоречит тому, что $T_{d}(u)$ асимптотически сохраняет меру.

Если $q \leqslant-2$, то числа $u_{0}=2^{n}-1$ и $u_{1}=2^{n}+2 q+2$ принадлежат множеству $\left\{0, \ldots, 2^{n}-1\right\}$ при любом достаточно большом натуральном $n$. Справедливо сравнение

$$
\frac{1}{2} u_{0}\left(u_{0}-d\right)=\frac{1}{2} u_{1}\left(u_{1}-d\right)\left(\bmod 2^{n}\right) .
$$

Это противоречит биективности полинома $T_{d}(u)$ по $\bmod 2^{n}$.

Рассмотрим случай $d=-1$. Из следствия 5.10 работы [3] вытекает, что полиномы $T_{-1}(2 u)$ и $T_{-1}(1+2 u)$ асимптотически сохраняют меру. В силу утверждения 2 из сравнения

$$
T_{-1}(2 u)-T_{-1}\left(1+2\left(2^{n}-1-u\right)\right)=2^{n}+2^{n+2} u-2^{2 n+1}=2^{n}\left(\bmod 2^{n+1}\right)
$$

следует, что полином $T_{-1}(u)$ асимптотически сохраняет меру. Это доказьвает теорему в одну сторону. Доказательство теоремы в обратную сторону очевидно. Теорема доказана.

В заключение автор выражает глубокую благодарность В. С. Анашину за постоянное внимание и поддержку в работе.

\section{СПИСОК ЦИТИРОВАННОЙ ЛИТЕРАТУРЫ}

[1] Кейперс Л., Нидеррейтер Г. Равномерное распределение последовательностей. М.: Наука, 1985.

[2] Анашин В.С. Равномерно распределенные последовательности целых $p$-адических чисел // Матем. заметки. 1994. Т. 55. № 2. С. 3-46.

[3] Anashin V. Uniformly Distributed Sequences over $p$-adic Integers. Preprint. Moscow: Russian State University for Humanities, 1993.

[4] Шафаревич И. Р. Основы алгебраической геометрии. Т. 1. М.: Наука, 1988.

[5] Хартсхорн Р. Алгебраическая геометрия. М.: Мир, 1981. 